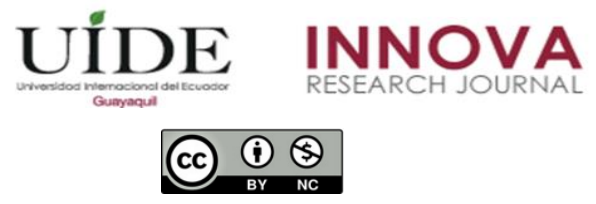

INNOVA Research Journal, ISSN 2477-9024

(Septiembre-Diciembre 2020). Vol. 5, No.3.2 pp. 111-121

DOI: https://doi.org/10.33890/innova.v5.n3.2.2020.1597

URL: http://revistas.uide.edu.ec/index.php/innova/index

Correo: innova@uide.edu.ec

\title{
Determinantes de la felicidad según los estudiantes de pre-grado durante el estado de emergencia sanitaria - Lima
}

\section{Determinants of happiness in undergraduate students during the state of health emergency - Lima}

\author{
Ronald Fernando Dávila Laguna \\ https://orcid.org/0000-0001-9886-0452 \\ Wuily Franz Trujillo Hinojosa \\ https://orcid.org/0000-0002-7610-6130 \\ Irma Milagros Carhuancho Mendoza \\ (D) https://orcid.org/0000-0002-4060-5667 \\ César Trujillo Hinojosa \\ (D) https://orcid.org/0000-0002-6684-5749 \\ Universidad César Vallejo, Perú
}

Autor para correspondencia: ronalddavilalaguna@gmail.com; wilfranztrujillo@gmail.com; imilagroscm@ucvvirtual.edu.pe; ctruhi@hotmail.com

Fecha de recepción: 05 de octubre de 2020 - Fecha de aceptación: 15 de diciembre de 2020

\begin{abstract}
Resumen
La investigación tuvo como objetivo establecer los determinantes de la felicidad según estudiantes de pre-grado durante el estado de emergencia sanitaria - Lima. Este se desarrolló bajo el enfoque cuantitativo, tipo sustantivo, diseño ex post facto, método hipotético deductivo. La población de estudio estuvo conformada por 1556 estudiantes, y participaron voluntariamente 308, para la recopilación de datos se aplicó una encuesta y cuestionarios on line de Escala de Felicidad para adultos de Moyano (2016), Inteligencia emocional de BarOn ICE: NA (2005) y de Engagement de Salanova y Schaufeli - UWES (Utrech Work Engagement Survey) (2016). Los datos se analizaron con el apoyo de la estadística descriptiva e inferencial con ecuaciones estructurales con el software SPSS 24 y Amos 24. Los resultados evidenciaron que predominó el nivel medio de la felicidad, el nivel alto de inteligencia emocional y engagement. Asimismo, el modelo teórico planteado fue que la inteligencia emocional y el engagement determinan la felicidad, por lo tanto, a partir de los resultados obtenidos con la prueba de Chicuadrado (131.804) y el nivel de significancia de .000, se aceptó el modelo. Finalmente, se demostró que la inteligencia emocional influyó en la felicidad en un $22 \%$, mientras que el engagement en un 36\%; asimismo la inteligencia emocional se relacionó con el engagement en un 33\%, en consecuencia la felicidad está determinada en un $23 \%$ por la inteligencia emocional y el engagement según los estudiantes de pre-grado durante el estado de emergencia sanitaria - Lima.

Palabras claves: felicidad; inteligencia emocional; engagement; estudiantes; pre grado.
\end{abstract}




\begin{abstract}
The research had the objective of establishing the determinants of happiness according to undergraduate students during the state of sanitary emergency - Lima. This was developed under the quantitative approach, substantive type, ex post facto design, hypothetical deductive method. The study population consisted of 1556 students and 308 participated voluntarily, for data collection an online Happiness Scale survey and questionnaires for adults in Moyano (2016), Emotional Intelligence de BarOn ICE: NA (2005) and Engagement de Salanova y Schaufeli UWES (Utrech Work Engagement Survey) (2016) were applied. The data were analyzed with the support of descriptive and inferential statistics with structural equations using SPSS 24 and Amos 24 software. The results showed that the medium level of happiness, the high level of emotional intelligence and engagement predominated. Likewise, the theoretical model proposed was that emotional intelligence and engagement determine happiness; therefore, from the result obtained with the Chicuadrado test $(131,804)$ and the significance level of .000 , the model was accepted. In conclusion, it was demonstrated that emotional intelligence influenced happiness by $22 \%$ while engagement by $36 \%$; likewise emotional intelligence was related to engagement by $33 \%$, in consequence happiness is determined in $23 \%$ by happiness and engagement according to undergraduate students during the state of health emergency - Lima.
\end{abstract}

Keywords: happiness; emotional intelligence; engagement; students; undergraduate.

\title{
Introducción
}

Desde la aparición del hombre sobre la tierra se interiorizaba en nuestro ser algún tipo de conceptualización en relación a la felicidad, independientemente de algunas emociones cotidianas las cuales a pesar del paso del tiempo no ha variado mucho, actualmente se tienen investigaciones, informes, reportes y artículos que tratan el tema con resultados variopintos, al respecto Helliwell, Layard, Sachs, y De Neve (2020) en el informe World Hapiness Report proponen una fórmula que permite tener ciudadanos felices.

En este sentido las instituciones del estado deben brindar servicios de calidad, desterrar la corrupción y generar confianza en su población; de otro lado Tanaka y Tokimatsu (2020) establecen que el ocio y la carrera profesional son factores que contribuyen a la felicidad, lo cual se complementa con lo planteado por Buettner, Nelson, y Veenhoven (2020) quienes establecen estrategias de política para alcanzar la felicidad, entre ellas invertir en investigación, apoyo a los integrantes de sociedades vulnerables y fortalecimiento del clima social, promoviendo el trabajo comunitario a través de las organizaciones no gubernamentales, por todo lo afirmado previamente se decidió que estudiar el constructor de la felicidad en estudiantes universitarios de ingeniería industrial de un programa para adultos en tiempos de estado de emergencia era preponderante.

\section{Problemática}

La felicidad es un constructo que se ha constituido desde épocas muy remotas hasta el presente, tanto para investigadores, personas de a pie, organizaciones y países, es tanta la controversia en su concepción y su medición, que ha pasado a ser un tema de relevancia para el poblador mundial. En el World Hapiness Report (2020) afirma que es necesario establecer 
determinantes culturales de la felicidad ciudadana que sirva de referencia para continuar desarrollando investigaciones Helliwell, Layard, Sachs, y De Neve (2020), asimismo es necesario precisar también que en Ways to Greater Happiness: A Delphi Study, concluyen que el crecimiento económico es una estrategia ineficaz para lograr la felicidad, y que ello debe reemplazarse por más inversión en investigar este constructo, fortalecimiento de las relaciones sociales, propiciar un buen gobierno e invertir en educación Buettner, Nelson, y Veenhoven (2020), esto concuerda con Ngoo, Tan y Tey (2020) quienes señalan que en el continente asiático la variable ingresos no tiene implicancia significativa en comparación con la calidad de vida, sin embargo en la mitad del continente la variable empleo si resultó significativo, de la misma manera Helliwell, Layard, Sachs, y De Neve, (2020) establecen como conclusión de su estudio Social Capital, Subjective Well-Being, and Happiness: Evidence from a Survey in Various European and Asian Countries to Address the Stiglitz Report que la felicidad tiene una correlación con el capital social, fortalecido a partir del empoderamiento de los lazos entre dichos actores sociales, utilizando herramientas como las redes sociales, también es preciso considerar lo que afirma Kennedy (2020) quien afirma que las personas que no toman en cuenta los estímulos futuros, se encuentran menos satisfechos y menos felices con la vida que aquellos que le dan un valor más significativo a dichos estímulos; siguiendo en la misma tendencia presentamos a Lee, Ha y Lee (2019) quienes establecieron cinco determinantes que presentan relación con la felicidad, que según los autores servirá de base para el fomento de políticas públicas a través de programas que coadyuven a la formación de estudiantes con identidad propia para la consecución de la felicidad.

A nivel de Latinoamérica se han desarrollado investigaciones para medir la felicidad en diferentes sectores de la población utilizando instrumentos anglosajones, lo cual no ha reflejado la cultura e idiosincrasia del poblador latino, ello ha diversificado de alguna manera los resultados obtenidos, ante este escenario es necesario destacar el interés de investigadores latinoamericanos por crear instrumentos que se ajusten a la realidad regional y tomen en cuenta el factor cultural, este aporte ha contribuido en el incremento en el desarrollo de investigaciones utilizando estas herramientas y se obtengan resultados acorde con la realidad latinoamericana, así tenemos a Alarcón (2006) con su escala de Felicidad de Lima (EFL), instrumento que consta de cuatro dimensiones y 27 ítems, ha Moyano, Dinamarca, Mendoza-llanos y Palomo-Vélez (2018) con la escala de felicidad para adultos (EFPA) de tres dimensiones y 21 ítems, ha Álvarez- Ramírez (2012) con un instrumento de 47 ítems denominado creencias acerca de la felicidad y también ha Rojas (2007) que creo el instrumento para identificar el referente de la felicidad, la cual consta de ocho referentes, estas investigaciones han contribuido a que la medición de la felicidad se desarrollen con instrumentos autóctonos que recogen el sentir del poblador latino.

En el Perú también se estudia la felicidad y por ejemplo se tiene a Pantigoso, De la Fuente, Torres, Arellano, y Sosa (2019) que han denominado Kusikuy a su estudio utilizando el lenguaje autoctono del país, que se traduce como estado de ánimo de bienestar o de felicidad más sostenido, en él se propone medir la felicidad implícita es decir aquella que manifiestan los ciudadanos para luego relacionarlos con factores materiales y sociales entre los que podemos mencionar bienes, servicios y familia. 
Los autores han tomado a la población de Lima donde se realizó el estudio como representativa de todo el Perú, ello manifiestan debido a que en esta ciudad se identificaron a pobladores de todos los orígenes, el resultado que obtienen es que las personas son relativamente felices, pues en un rango del 0 al 20 escala propuesta para medir la felicidad se obtuvo 16 que representa "un más o menos con tendencia hacia bien".

Así mismo, concluyen que los niveles antagónicos tanto en edad como en riqueza muestran signos de mayor felicidad, es decir los más jóvenes y adultos mayores al igual que los más ricos y pobres presentan signos de mayor felicidad que aquella población que se encuentra en la parte intermedia de dicha escala.

Otro resultado interesante a tomar en cuenta es que la felicidad individual del peruano es sentida en mayor dimensión que la social ello se debe según el estudio a la falta de identificación de cada individuo con su grupo y además al resultado de mostrar a través de la comunicación social lo malo que pasa en nuestra sociedad.

Sin embargo, tal como manifiestan Pantigoso, De la Fuente, Torres, Arellano, y Sosa (2019) existe un resultado cuyo aporte es mayor y que es la aparente paradoja entre la felicidad individual y la felicidad social, luego de un análisis profundo se determina que tal felicidad-la individual-lo logran en aspectos ligados a la satisfacción familiar individual y a lo relacionado al consumo.

Es decir los pobladores peruanos hallan bienestar cuando consiguen algo debido a sus propios medios, concepto "self made natión". Llama también a la reflexión que se haya determinado que la solidaridad no sea una de las variables más importantes para la felicidad de los peruanos, así como una relación amigable con el medio ambiente, finalmente el estudio hace mención que estamos ante el inicio de un cambio en la que la solidaridad con el entorno social y ambiental empezará a tomar mayor importancia, para ello proponen la realización de investigaciones que coadyuven a la consecución de este escenario, es decir si como país solo queremos el desarrollo o si queremos ser un país Kusikuy, un país de gente feliz Pantigoso, de la Fuente, Torres, Arellano, y Sosa (2019).

Realizando un enfoque en un entorno local, se ha podido observar, que la población de estudio a pesar del estado de emergencia en que se vive por la pandemia, y ante la necesidad de generar ingresos que solventen la canasta familiar, se ven en la necesidad de ejercer su actividad cotidiana en forma completa priorizando de esta manera su actividad laboral.

El entorno laboral en el cual se desempeña y más aún por la coyuntura actual en que se vive, ha hecho que en de dicha actividad laboral se establezcan estándares mínimos de cumplimiento obligatorio no solo laboral sino también de seguridad sanitaría, que es indispensable interiorizar y cumplir si se quiere ser competitivos y mantenerse vigente, lo cual conlleva a destinar recursos adicionales no previstos inicialmente, que es necesario restar del destinado a la familia. 
Por lo absorbente en términos de tiempo que resulta desempeñar la actividad laboral por la alta responsabilidad que implica el desarrollo de tareas propio de la mano de obra calificada, conlleva a que se reduzca las horas destinadas a fortalecer los lazos familiares, lo cual se refleja en el ritmo de vida acelerado en la que el tiempo pasa "volando" y se nos va literalmente de las manos, lo cual va debilitando los cimientos de la estructura familiar que anteriormente se encontraba fortalecida.

Lo detallado anteriormente va debilitando sistemáticamente los lazos de calor y calidez familiar lo cual repercute negativamente en las relaciones familiares causando con sutileza la carencia de apoyo por parte de los integrantes de la familia, si a ello se suma la confinación de sus integrantes, el alto riesgo de asistir al trabajo presencial y en algunos casos el trabajo remoto, notaremos que el aspecto socio emocional del individuo se deteriora lo cual puede ser motivo para generar el estrés en el seno familiar y en el caso del estudiante universitario el estrés laboral .

Así mismo, es preciso señalar que en esta coyuntura el aspecto económico ha golpeado muy fuertemente a los estratos sociales más vulnerables y por añadidura a la población objeto de estudio lo cual repercute en la tranquilidad del individuo, todo lo dicho si se integran es suficiente razón para generar altos niveles de estrés laboral, con esta enfermedad interiorizado en su persona, el estudiante universitario puede trasladar esto a su entorno familiar lo que provocaría un entorno crítico que afectaría la salud familiar.

Por las razones expuestas y ante la diversidad de enfoques existente en torno a la felicidad es que se pretende determinar si existen determinantes que incidan en la felicidad desde el punto de vista de estudiantes universitarios de un programa de formación para adultos

En cuanto al aspecto metodológico la presente investigación se debe de desarrollar debido a que se pretende utilizar un análisis multivariante cuyo diseño es innovador para nuestro medio, basado en las ecuaciones estructurales que permiten conocer cómo influyen y se relacionan las diversas variables independientes y dependientes identificadas para esta investigación, cumpliendo de esta manera con sus objetivos.

\section{Metodología}

Para definir el enfoque del estudio se tomó en cuenta a Carhuancho, Nolazco, Sicheri, Guerrero, y Casana (2019) que respecto al enfoque cuantitativo manifiestan que este se nutre de las ciencias aplicadas y utiliza herramientas matemáticas para su análisis, lo cual se complementa con lo afirmado por Hernández, Fernández y Baptista (2010) que además señalan que es factible generalizar los resultados producto de ejercer algún control sobre los fenómenos, también mide variables y se pueden utilizar desde investigaciones exploratorias hasta experimentales (Niño, 2011); por lo mencionado líneas arriba se confirma que el enfoque cuantitativo es el que más se ajusta por su preponderancia y ajuste a la realidad de estudio. 
En cuanto al tipo de estudio, se afirma que si se tiene una orientación esencialmente dirigida a conocer los fenómenos a través de la descripción y la explicación de los hechos, estaremos identificando a una investigación sustantiva Sánchez, Reyes, Mejía (2018); ello está en la misma línea con lo manifestado por Sánchez y Reyes (2015) los cuales afirman que, desde el instante en que una investigación se dirige a la búsqueda de la verdad para responder a los problemas suscitados y de alguna manera está encaminada a un estudio básico se denomina sustantiva, lo presentado es muy relevante para nuestro estudio pues deslinda la ambigüedad generada por la elección entre tipos de investigación básica y aplicada y ayuda a aclarar el panorama.

Con respecto al diseño esta es ex post facto, ello basado en Hernández, Fernández, y Baptista (2010) quienes afirman que, cuando se investiga la realidad y en estos ya ocurrieron los hechos y se suscitaron las consecuencias, entonces estamos hablando de este tipo de diseño y lo que hace el investigador es observar y dar a conocer los resultados, en el mismo rumbo se encontró a Sánchez, Reyes, Mejía (2018) que establecen, desde el momento en que pretendemos explicar los resultados de un acontecimiento o suceso ya ocurrido estaremos frente a una investigación cuyo diseño corresponde a un ex post facto, así mismo se ubicó a Rodríguez y Valldeoriola (2014) quienes manifiestan lo mismo pero con un componente adicional, cuando se trata de estudios en que el investigador no controla ninguna variable independiente debido a que el suceso que se está estudiando ya ocurrió entonces el diseño será correlacional con modelo de ecuaciones estructurales.

Acerca de método elegido para el estudio, se afirma que, desde el instante en que se usan procesos lógicos deductivos en la ruta del conocimiento y respetando el procedimiento científico a partir de un supuesto el cual debe de ser demostrable se estará hablando del método hipotético-deductivo Sánchez, Reyes y Mejía (2018), esta afirmación concuerda con lo planteado por Sánchez (2019), quien ademas adiciona, que debe tener una orientación sutil a aumentar la teoría y a solucionar problemas tanto teóricos como prácticos, también se encontró a Rodríguez y Pérez (2017), quien establece que partiendo de plantear una hipótesis producto del análisis de principios, leyes o datos empíricos se arriba a deducciones predictivas previamente sometidas a verificación y si se establece la respectiva correspondencia fáctica se estará definiendo al método hipotético deductivo, de todo lo analizado se puede establecer que el estudio tiene una concepción clara del método que permitió obtener resultados coherentes que explican los objetivos planteados.

En lo concerniente a la muestra se determinó que fueran 308 individuos obtenidos de una población de 1556 estudiantes de un programa de formación para adultos, a los cuales se aplicó una herramienta estandarizada tipo cuestionario, basado en la técnica de la encuesta, para ello se utilizó el muestreo probabilístico, utilizando el método muestreo simple aleatorio PimientaLastra (2000), para el cálculo del tamaño de la muestra se utilizó la fórmula para población finita especificado por Aguilar-Barojas (2005) bajo los siguientes parámetros: p=0,50; q=0,50; e=5\% y $\mathrm{Z}=1,96$.

\section{Instrumento: Escala de Felicidad Para Adultos (EFPA)}


En cuanto al instrumento seleccionado, escala de felicidad para adultos (EFPA) Moyano, Dinamarca, Mendoza, Palomo (2018), se eligió luego de una búsqueda exhaustiva y tomando en cuenta sus tres dimensiones a saber: a) equilibrio psicológico, b) satisfacción familiar y (c) orientación de logro, la cual considera las características de la población en estudio, la EFPA establece 21 items, de ellos los nueve primeros tienen una escala tipo Likert de 6 puntos donde 1 corresponde a "Totalmente de acuerdo" y 6 a "Totalmente en desacuerdo" por el tipo de pregunta, luego desde la pregunta 10 hasta la 21 con la misma escala, con 1 referido a "Totalmente en desacuerdo" y 6 a "Totalmente de acuerdo" cuyos ítems se han distribuido de la siguiente manera:

\section{Tabla 1}

Items según dimensión para el instrumento EFPA

\begin{tabular}{lc}
\hline Dimensión & \multicolumn{1}{c}{ Ítem } \\
\hline Equilibrio Psicológico & $1,2,3,4,5,6,7,8$ y 9 \\
Satisfacción familiar & $10,11,12,13,14$ y 15 \\
Orientación de logro & $16,17,18,19,20$ y 21 \\
\hline
\end{tabular}

Tabla 2

Coeficiente de consistencia interna para el instrumento EFPA

\begin{tabular}{llll}
\hline & Estado Psicológico & Tener Familia & Orientación de Logro \\
\hline Alfa de Cronbach & 0.911 & 0.927 & 0.904 \\
Alfa Ordinal & 0.936 & 0.965 & 0.946 \\
Omega & 0.916 & 0.957 & 0.931 \\
Theta & 0.920 & 0.960 & 0.930 \\
$\mathrm{~N}^{\circ}$ de Ítems & 9 & 6 & 6 \\
\hline
\end{tabular}

Fuente: Moyano, Dinamarca, Mendoza y Palomo, (2018)

Theta asume un análisis factorial (método de componentes principales), en tanto Omega es una medida de fiabilidad de una prueba de homogeneidad basada en el modelo de un solo factor, por lo que es especialmente adecuada para evaluar la fiabilidad de cada uno de los tres factores de la escala. Aun cuando el Alfa de Cronbach es más bajo en todos los factores que el Alfa Ordinal, las variaciones generales están entre 0.904 (logro) y 0.965 (tener familia). 
Para el análisis de los datos se referencia a Sánchez, Reyes y Mejía (2018), quienes afirman, el análisis de la información se constituye en una etapa del sistema de estudio que consiste en armonizar los datos recogidos para que pueda ser evaluada de manera detallada y analítica, describiendo, caracterizando e interpretando la información.

Este análisis se puede realizar de manera cualitativa o cuantitativa, o de ambas formas, lo cual se puede ampliar mediante el uso de las ecuaciones estructurales, que se han constituido en una técnica que interrelaciona tanto la regresión múltiple como el análisis factorial. Lo cual sirvió como cimiento para decidir por el uso de esta herramienta para el análisis de datos en este estudio, que también a la par confirma lo establecido mediante Cupani (2012), refrendado por Medrano y Muñoz-Navarro (2017), quienes establecen que el análisis mediante Ecuaciones Estructurales (Structural Equation Modeling; SEM), se transforman en la técnica más poderosa y conveniente para examinar la integridad de una dependencia causal, a pesar de que se utilicen diseños no experimentales. La clave de este método radica en estudiar la posibilidad de un direccionamiento causal y reemplazar la inspección experimental por una inspección estadística, propiciando de esta manera un seudoaislamiento de las variables en estudio.

\section{Tabla 3}

Hipótesis establecidas

\begin{tabular}{ll}
\hline Hipótesis & Descripción \\
\hline $\mathrm{H}_{1}$ & La inteligencia emocional tiene influencia positiva en la felicidad de los estudiantes \\
& universitarios de pre grado en Lima \\
$\mathrm{H}_{2}$ & El engagement influye positivamente en la felicidad de los estudiantes universitarios \\
& de pregrado en Lima. \\
$\mathrm{H}_{3}$ & La inteligencia emocional tiene relación positiva con el engagement y determinan la \\
& felicidad de los estudiantes universitarios de pregrado en Lima \\
\hline
\end{tabular}

\section{Resultados}

De acuerdo a las hipótesis planteadas en la tabla 3, se utilizó para el análisis de los datos un modelo que se ajuste al número y comportamiento de estos, siendo las ecuaciones estructurales la elegida, para el procesamiento se empleó el software SPSS 24 y Amos 24 las cuales nos permitieron obtener información descriptiva y correlacional de las variables analizadas, con lo cual se puede determinar la influencia e interrelación directa e indirecta entre las variables en forma simultánea, tal como se puede apreciar en la figura 1.

Para validar el modelo utilizado es necesario contar con indicadores Vergara, Quezada y Blanco (2011) quienes afirman que además del $X^{2}$, P-value, se debe contar con la razón $X^{2} / d f$, el índice NFI (índice de ajuste normado), en el caso de la razón $X 2 / d f$ se recomiendan valores inferiores a 4; para el índice NFI se recomiendan cifras superiores a 0.90; para el RMSEA Browne y Cudeck (1993) citado por Morata-Ramírez, Holgado-Tello, Barbero-García y Méndez 
(2015) establece que valores menores a 0.05 indican un buen ajuste y valores comprendidos entre 0.05 y 0.08 un ajuste razonable.

Los indicadores seleccionados para validar el modelo se presentan en la tabla 5

\section{Tabla 5}

Indicadores que validan el modelo

\begin{tabular}{ll}
\hline Indicador & Valor \\
\hline Chi-Square $\left(\mathrm{X}^{2}\right)$ & 131.804 \\
Degress of Freedom $(\mathrm{df})$ & 41 \\
P-value & 0.000 \\
$X^{2} / d f$ & 3.21 \\
NFI & 0.89 \\
RMSEA & 0.08 \\
\hline
\end{tabular}

\section{Figura 1}

Variables independientes y dependientes, sus influencias y relaciones

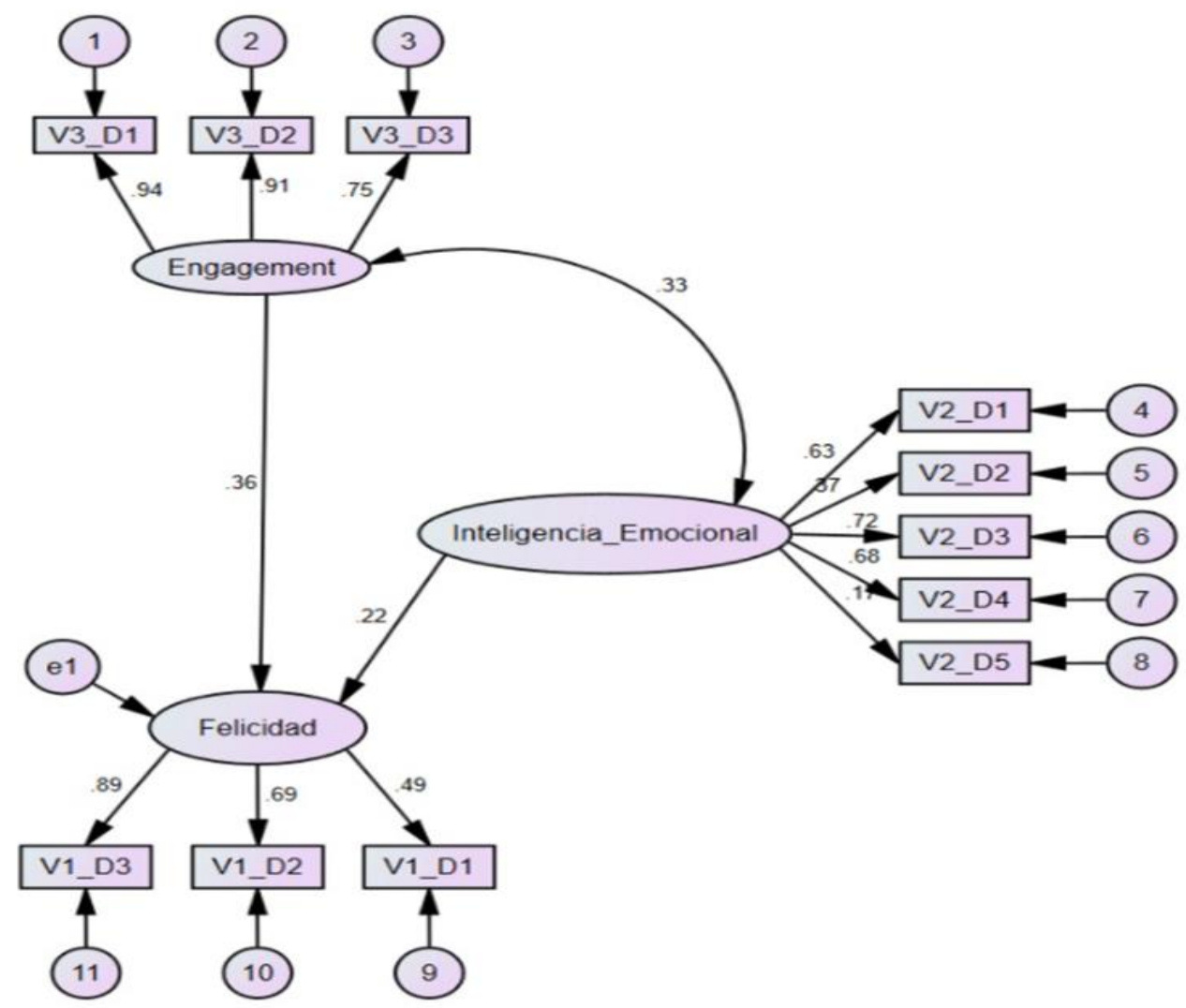

Chi-Square $=131.804, \mathrm{df}=41, \mathrm{P}-$ value $=0.000, \mathrm{RMSEA}=0.08$

*Resultado gráfico arrojado por el software Amos 24. Las figuras elípticas representan las variables analizadas y los rectángulos las dimensiones de cada variable en forma sistemática. 
De lo observado en la Figura 1 se obtienen evidencias de que existe influencia directa de la variable Inteligencia Emocional y Engagement ambas independientes sobre la variable dependiente Felicidad con 0.22 y 0.36 , así mismo se presenta una correlación entre las variables independientes de 0.33 las cuales se relacionan con la felicidad.

\section{Conclusiones}

Producto del proceso investigativo desarrollado y de acuerdo a los resultados obtenidos se afirma que existe influencia de la Inteligencia Emocional y del Engagement sobre la Felicidad según los estudiantes universitarios de pregrado de un programa de formación para adultos de la carrera profesional de ingeniería industrial.

También queda demostrada que las variables independientes presentan una interrelación estadísticamente significativa lo cual determina la felicidad en la población bajo estudio. Así mismo es preciso señalar que la dimensión equilibrio psicológico tiene estadísticamente mayor influencia significativa sobre la felicidad.

El presente estudio sirve para aperturar los umbrales de la investigación sobre la felicidad en otros sectores de la población tomando en cuenta otras variables que estén relacionados con la actividad laboral de la población bajo estudio.

\section{Referencias Bibliográficas}

Aguilar-Barojas, S. (2005). Fórmulas para el cálculo de la muestra en investigaciones de salud. Salud en Tabasco, 333-338. Obtenido de https://www.redalyc.org/pdf/487/48711206.pdf

Alarcon, R. (2006). Desarrollo de una Escala Factorial para Medir la Felicidad. Revista Interamericana de Psicología/Interamerican Journal of Psychology, 99-106.

Alvarez-Ramirez, L. (2012). Escala de creencias acerca de la felicidad población adulta de la ciudad de Bucaramanga (Colombia). Investigación \& Desarrollo, 302-333. Obtenido de https://www.redalyc.org/pdf/268/26824854004.pdf

Buettner, D., Nelson, T., \& Veenhoven, R. (2020). Ways to Greater Happiness: A Delphi Study. Journal of Happiness Studies.

Carhuancho, I., Nolazco, F., Sicheri, L., Guerrero, M., \& Casana, K. (2019). Metodología para la investigación holistica. Guayaquil: UIDE.

Helliwell, J., Layard, R., Sachs, J., \& De Neve, J. (2020). World Happiness Report 2020. Estados Unidos: Columbia University.

Hernández, R., Fernández, C., \& Baptista, M. (2010). Metodología de la Investigación. México: Mc Graw-Hill.

Kennedy, J. (2020). Subjective Wellbeing and the Discount Rate. J Happiness Stud 21, 635-658.

Lee, H., Ha, J., \& Lee, H. (2019). Happiness: Identifying Important Determinants of Happiness from University Students. View Point. Ji Kyung-Yeon, 137-153. 
Medrano, L., \& Muñoz-Navarro, R. (2017). Aproximación conceptual y práctica a los Modelos de Ecuaciones Estructurales. Revista Digital de Investigación en Docencia Universitaria, 219-239.

Morata-Ramirez, A., Holgado-Tello, F., Barbero-García, I., \& Mendez, G. (2015). Análisis factorial confirmatorio. Recomendaciones sobre mínimos cuadrados no ponderados en función del error tipo I de Ji-Cuadrado y RMSEA. Acción Psicológica, 79-90. doi:dx.doi.org/10.5944/ap.12.1.14362

Moyano, E., Dinamarca, D., Mendoza, R., \& Palomo, G. (2018). Escala de Felicidad para Adultos (EFPA). Terapia Psicológica, 33-45.

Ngoo, Y., Tan, E., \& Tey, N. (2020). Determinants of Life Satisfactión in Asia: A Quantile Regression Approach. J Happiness Stud.

Niño, V. (2011). Metodología de la investigación. Diseño y ejecución. Bogotá: Ediciones de la U.

Pantigoso, P., De la Fuente, L., Torres, L., Arellano, R., \& Sosa, M. (s.f.). Kusikuy: La felicidad de los peruanos.

Pimienta Lastra, R. (2000). Encuestas probabilísticas vs. no probabilísticas. Política y cultura, 263-276. Obtenido de https://www.redalyc.org/pdf/267/26701313.pdf

Rodríguez, A., \& Pérez, A. (2017). Métodos científicos de indagación y de construcción del conocimiento. Revista Escuela de Administración de Negocios, 1-26.

Rodríguez, D., \& Valldeoriola, J. (2014). Metodología de la investigación . Catalunya: Universidad Oberta de Catalunya.

Salanova, M., \& Schaufeli, W. (2016). El engagement de los empleados: Un reto emergente para la direccion de los recursos humanos. ResearchGate, 109-138. Obtenido de https://www.researchgate.net/publication/46650776

Sánchez, F. (2019). Fundamentos Epistémicos de la Investigación Cualitativa y Cuantitativa: Consensos y Disensos. Revista Digital de Investigación en Docencia Universitaria, 102122.

Sánchez, H., \& Reyes, C. (2015). Metodología y diseños en la investigación científica. Lima: Editorial Business Suport Aneth SRL.

Sánchez, H., Reyes, C., \& Mejía, K. (2018). Manual de términos en investigación científica, tecnológica y humanista. Lima: Universidad Ricardo Palma.

Tanaka, S., \& Tokimatsu, K. (2020). Social Capital, Subjective Well-Being, and Happiness: Evidence from a Survey in Various European and Asian Countries to Address the Stiglitz Report. Scientific Research Publishing, 322-348.

Ugarriza, N., \& Pajares, L. (2005). La evaluación de la inteligencia emocional a través del inventario de Baron ICE: NA, en una muestra de niños y adolescentes. Persona, 11-58.

Vergara, J., Quesada, V., \& Blanco, I. (2011). Análisis de la calidad en el servicio y satisfacción de los usuarios en dos hoteles cinco estrellas de la ciudad de Cartagena (Colombia) mediante un modelo de ecuaciones estructurales. Ingeniare. Revista chilena de ingeniería, 420-428. Obtenido de https://scielo.conicyt.cl/pdf/ingeniare/v19n3/art11.pdf 